\title{
Neuromielitis óptica. Principales diferencias con la esclerosis múltiple
}

\author{
I. LOPATEGUI CABEZAS, M. CERVANTES LLANO ${ }^{1}$, G. PENTÓN ROL ${ }^{2}$ \\ Departamento de Bioquímica. Instituto de Ciencias Básicas y Preclínicas "Victoria de \\ Girón”. 'Ciencias Farmacéuticas. ${ }^{2}$ Centro de Ingeniería Genética y Biotecnología. \\ Ciudad de La Habana. Cuba
}

\section{RESUMEN}

La neuromielitis optica o síndrome de Devic es una enfermedad inflamatoria, desmielinizante y autoinmune del sistema nervioso central. Se caracteriza por ataques de neuritis óptica y mielitis, pudiendo producir ceguera, gran invalidez neurológica e incluso la muerte a corto plazo. Hasta el momento no existe un tratamiento efectivo, la terapia se centra en el tratamiento de los ataques agudos, la prevención médica de las complicaciones y la rehabilitación. Se realiza una revisión de esta enfermedad poco común, considerando que su prevalencia en nuestro país ha ido en aumento y se establece una comparación entre la neuromielitis óptica y la esclerosis múltiple, basándose en las principales características clínico-epidemiológicas, que distinguen estas dos patologías, consideradas por muchos variantes clínicas de una misma enfermedad.

PALABRAS CLAVES: Neuromielitis óptica. Devic. Esclerosis múltiple. Autoinmunidad.
OPTIC NEUROMYELITIS. MAIN DIFFERENCES WITH MULTIPLE SCLEROSIS

\section{INTRODUCCIÓN}

La neuromielitis óptica (NMO) o síndrome de Devic, enfermedad inflamatoria, autoinmune y desmielinizante del sistema nervioso central (SNC) se caracteriza por ataques de neuritis óptica (NO) y mielitis, provoca gran invalidez neurológica, ceguera y hasta la muerte, estos eventos clínicos pueden ocurrir también en la esclerosis múltiple (EM), pero en la NMO son por lo general más agudos y severos $(1,2)$.

La NMO tiende a seguir un curso monofásico o recidivante, es decir, por brotes o recaídas (más del $70 \%$ de los casos tienen un curso recidivante). En el monofásico los pacientes experimentan una NO unilateral o bilateral y un episodio de mielitis, con un corto período de tiempo entre ellos y sin recaídas posteriores. Sin embargo, los pacientes con un curso recidivante continúan teniendo exacerbaciones de NO y/o mielitis (3).

Diferentes pruebas apoyan el diagnóstico de la NMO:

-Resonancia magnética nuclear (RMN) de cerebro y médula espinal: el diagnóstico de NMO se basa en la ausencia

\begin{abstract}
The optic neuromyelitis or syndrome of Devic is an inflammatory and autoimmune illness of the central nervous system. It is characterized by attacks of optic neuritis and myelitis, being able to produce blindness, great neurological disability and even the short term death. Until the moment an effective treatment doesn't exist, the therapy is centred in the treatment of the acute attacks, the medical prevention of the complications and the rehabilitation. This article is a revision of this not very common illness, considering that its prevalence in our country has gone in increase. We compare between the optic neuromyelitis and the multiple sclerosis, being based on the main ones characteristic clinical-epidemic that distinguishes these two pathologies, considered by many clinical variants of oneself illness.
\end{abstract}

KEY WORDS: Optic neuromyelitis. Devic. Multiple sclerosis. Autoimmunity. de lesiones parenquimatosas cerebrales (excluyendo los nervios ópticos), la RMN de médula espinal muestra signos de alteraciones en T2 que se extiendan sobre tres o más segmentos vertebrales. En la EM las lesiones inflamatorias desmielinizantes se localizan en el cerebro $(4,5)$.

-Estudio del líquido cefalorraquídeo (LCR): los pacientes con NMO tienen una pleiocitosis de más de 50 leucocitos $/ \mathrm{mm}^{3}$, fundamentalmente en los períodos de exacerbación de la mielitis aguda, el diferencial de leucocitos en el LCR de los enfermos revela la presencia de neutrófilos, estas anormalidades pueden reflejar la severidad de la mielitis, la cual a menudo desemboca en la necrosis (6).

Aproximadamente el $85 \%$ de los pacientes con EM tienen bandas oligoclonales que se detectan en la electroforesis del LCR. Contrariamente a esto, las bandas oligoclonales se presentan solo en el 15-35\% de los enfermos con NMO. Alteraciones de la inmunoglobulina $\mathrm{G}$, tales como incremento en la velocidad de su síntesis, pueden aparecer en la NMO, aunque menos comúnmente que en la EM $(6,7)$. 
-Exámenes serológicos: uno o más autoanticuerpos, incluyendo anticuerpos antinucleares, anticuerpos anti ADN de doble cadena y anticuerpos anti-tiroideos, así como antígenos nucleares extraíbles frecuentemente se encuentran al diagnosticarse la NMO $(8,9)$.

-Exámenes neurofisiológicos: los potenciales evocados son potenciales eléctricos generados en el SNC tras la estimulación de un órgano sensitivo/sensorial periférico. Pueden usarse para evaluar la conductividad de los impulsos eléctricos a través de fibras nerviosas mielinizadas. Los que se estudian con más frecuencia en la NMO son los visuales, que pueden ocasionalmente detectar lesiones subclínicas del nervio óptico (10).

Recientemente Wingerchuk y cols. han propuesto criterios para el diagnóstico de la NMO (11). Estos criterios fueron precedidos por los criterios de Mandler en 1993 (3) y de O'Riordan en 1996 (12).

Criterios de Wingerchuk y cols.

Criterios absolutos (El diagnóstico requiere todos los criterios absolutos).

1. Neuritis óptica.

2. Mielitis aguda.

3. Ausencia de enfermedad clínica fuera de la médula espinal y los nervios ópticos.

Criterios principales de soporte:

1. La RMN de cerebro debe ser negativa al diagnosticarse la enfermedad (normal o que no se encuentren criterios radiológicos de EM).

2. RMN de médula espinal con signos de alteraciones en T2 que se extiendan sobre tres o más segmentos vertebrales.

3. Pleiocitosis del LCR (más de 50 leucocitos $/ \mathrm{mm}^{3}$ ó más de 5 neutrófilos $/ \mathrm{mm}^{3}$ ).

Criterio secundario de soporte:

1. Neuritis óptica con agudeza visual fijada peor que 20/200 en al menos un ojo.

En Europa, donde la EM tiene una prevalencia de 100200/100.000 habitantes, la NMO se considera una enfermedad rara (12). Ambas enfermedades afectan fundamentalmente a los adultos jóvenes, preferentemente el sexo femenino y han sido reportadas en la infancia. Para la NMO la edad media de comienzo es entre 35 y 47 años y la mayoría de los reportes sugieren una relación hombre: mujer de aproximadamente 1:4 a $1: 8(11,12)$.

La NMO parece ser más común en los individuos no caucasianos tales como africanos, americanos, japoneses y de otras islas del Pacífico (13). El 7,6\% de los pacientes japoneses con EM y más del $6 \%$ de los casos de enfermedades desmielinizantes en la India padecen $\mathrm{NMO}(14,15)$

En Cuba la prevalencia de la NMO se desconoce. En los últimos años se ha observado un incremento en el número de pacientes diagnosticados con la enfermedad. La prevalencia de la EM también ha ido aumentando, siendo actualmente de 10/100.000 habitantes (16).

El papel de los factores genéticos no se conoce con profundidad. El análisis de la susceptibilidad genética, tanto en NMO como en EM, se ha basado principalmente en estudios de asociación de genes candidatos, en los cuales la frecuencia de marcadores alélicos es comparada en pacientes y controles. Ciertos alelos de antígenos leucocitarios humanos (HLA) se han encontrado asociados; en un estudio realizado el HLADPB1*0501 se relacionó con mayor frecuencia con la NMO y el HLA-DR2B con la EM (17).

\section{ASOCIACIONES DE OTRAS PATOLOGÍAS CON LA NMO}

Existen numerosos reportes de enfermedad óptico-espinal asociada con enfermedades del tejido conectivo y otras enfermedades autoinmunes, fundamentalmente con la EM (18-20). Pacientes japoneses con EM óptico-espinal tienen similar comportamiento a los pacientes con NMO recidivantes y difieren inmunogenéticamente de los japoneses con EM del oeste, que son HLA-DR2 negativos (14). Los aborígenes en Manitota: Canadá tienen un riesgo incrementado para diferentes enfermedades que han sido implicadas más en la patogénesis de la NMO que de la EM (18), entre estas se encuentran las infecciosas (21-23) enfermedades del colágeno, los síndromes de auto-anticuerpos y las exposiciones a agentes tóxicos (9).

Existen diferentes reportes de la asociación de la NMO con el lupus eritematoso sistémico (24). Algunos autores sugieren que exista una relación de los anticuerpos anticardiolipinas y los del lupus anticoagulante (25). La NMO también se ha encontrado asociada con el Síndrome de Sjogren (SS). Existen reportes de casos (26) en los que los anticuerpos antiRo SSA (Sjogren síndrome antígen A) fueron detectados en 7 de 11 pacientes con neuropatía asociada a SS. Los autores se refirieron a una reactividad cruzada entre Ro y $\mathrm{HuD}$, los antígenos asociados con la encefalomielitis paraneoplásica y la neuronopatía sensorial. La NMO fue también descrita en un paciente con anticuerpos citoplasmáticos antineutrófilos perinucleares (p-ANCA), anticuerpos antinucleares (ANA) y anticuerpos anti antígeno del SS (SSA y SSB) (27).

La NMO se ha asociada con enfermedades infecciosas como las virales, ya que frecuentemente tiene pródromos caracterizados por cefalea, mialgias y síntomas respiratorios altos. Múltiples organismos infecciosos se han propuesto como agentes causantes o co-factores en el desarrollo de la enfermedad, como la mononucleosis infecciosa aguda (21), la varicela-zoster (22) y las infecciones por HIV-1 (23).

Se manifiesta una alta frecuencia de alteraciones en la RMN en el eje hipotálamo-pituitario en pacientes con NMO y endocrinopatías asociadas. Vernant y cols. describieron una serie de 8 mujeres de Martinica y Guadalupe, 7 de las cuales tenían una amenorrea secundaria que coincidía con exacerbaciones de NMO. Todas las pacientes sufrieron de NO recurrente y mielitis que fue resistente a la terapia inmunosupresora y finalmente provocó ceguera y paraplejia. En este estudio los hallazgos neuropatológicos demostraron una pronunciada necrosis con desmielinización limitada, similar a lo reportado en la NMO (28).

\section{TRATAMIENTO}

Todas las recomendaciones terapéuticas en la NMO representan experiencias anecdóticas de una pequeña serie de casos no controlados. La terapia se centra en el tratamiento de los ataques agudos, la prevención médica de las complicaciones y la rehabilitación.

La mayoría de los pacientes que presentan NMO con exacerbaciones reciben tratamiento corticosteroide intravenoso (29). La plasmaféresis ha sido reportada como efectiva en el manejo de las crisis agudas, con o sin trastornos del tejido conectivo asociado (30). Esta intervención reduce el número de auto-anticuerpos circulantes y complejos inmunes lo cual 
puede explicar su efectividad en algunos pacientes con esta enfermedad. Por otra parte, la inmunoglobulina intravenosa también ha sido usada anecdóticamente.

Los ataques agudos de la médula cervical pueden causar paro respiratorio por lo que los pacientes con alto riesgo para esta complicación requieren de la hospitalización en una Unidad de Cuidados Intensivos.

La terapia preventiva se requiere en pacientes con enfermedad recidivante. La mayoría de las pacientes con NMO de Norteamérica reciben tratamiento con IFN- $\beta$ parenteral. Algunos médicos, basados en el hecho que no existe una experiencia controlada, creen que este tratamiento es inefectivo. Sin embargo, en un estudio japonés reciente el IFN $\beta$-1b mostró efectos beneficiosos (31).

En la EM existen fármacos aprobados por la FDA, el Interferon Beta-1b (Betaseron) en 1993, Interferon Beta-1a (Avonex) en 1996, Copaxone (Acetato de Glatiramer o copolímero 1) en 1996 y el recién aprobado Natalizumab, que son altamente costosos y son efectivos en reducir el $30 \%$ de las crisis en el caso del IFN beta y el Copaxone y el 66\% en el caso del Natalizumab (32).

En el único estudio de tratamiento prospectivo publicado, Mandler et al. encontraron que 7 pacientes con NMO de reciente diagnóstico se estabilizaron por al menos 18 meses con un régimen de Azatioprina y Prepnisona oral (33).

\section{Bibliografía}

1. Weinshenker B. Western vs Optic Spinal MS. Two diseases, one treatment? Rev Neurol 2005; 64: 594-595.

2. De Seze. Borderlines types of Multiple Sclerosis. Rev Neurol 2006; 162: 137-43.

3. Mandler RN, Davis LE, Jeffery DR, Kornfeld M. Devic's neuromyelitis optica: a clinicopathological study of 8 patients. Ann Neurol 1993; 34: $162-8$.

4. Rocca MA, Acosta F, Mezzapesa DM, Martinelli V, Salvi F, Ghezzi A, et al. Magnetization transfer and diffusion tensor MRI show gray matter damage in Neuromyelitis Optica. Neurol 2004; 62: 476-478.

5. Filippi M, Rocca MA, Moiola L, et al. MRI and magnetization transfer imaging changes in the brain and cervical cord of patients with Devic's neuromyelitis optica. Neurol 1999; 53: 1705-1710.

6. Bergamaschi R, Tonietti S, Franciotta D, Candeloro E, T avazzi E, Piccolo G, et al. Oligoclonal bands in Devic's neuromyelitis Optica and Multiple Sclerosis: Differences in repeated cerebrospinal fluid examinations. Mult Scler 2004; 10: 2-4.

7. Nakashima I, Fujihara K, Fujimori J, Narikawa K, Misu T, Itoyama Y. Absence of IgG1 response in the cerebrospinal fluid of relapsing neuromyelitis optica. Neurology 2004; 62: 144-6.

8. Lennon VA, Weinshenker BG. Identification of a marker autoantibody of Neuromyelitis Optica. Neurol 2003; 60 (5 Suppl 1): A519.

9. Lindsey LW, Albers GW, Steinman L. Recurrent transverse myelitis, myasthenia gravis, and autoantibodies. Ann Neurol 1992; 32: 407- 9.

10. Santos MA, Lana MA, Lei MS, Varella de A. Avaliaçäo dos potenciais evocados auditivos do tronco encefálico na esclerose múltipla / Auditory evoked potentials in multiple sclerosis. Arq Neuropsiquiatr 2003; 61(2B): 392-397.

11. Wingerchuk DM, Hogancamp W, O'Brien P. The clinical course of neuromyelitis optica (Devic's syndrome). Neurology 1999; 53: 110714.

12. O'Riordan JI, Gallagher HL, Thompson AJ, et al. Clinical, CSF, and MRI findings Devic's neuromyelitis optica. J Neurol Neurosurg Psychiatr 1996; 60: 382-7.

13. El Otmani H, Rafai MA, Moutaouakil F, El Moutawakkil B, Boulaajaj F Z, Moudden M, et al. Devic's neuromyelitis optica in Morocco. A study of 9-cases. Rev Neurol 2005; 161: 1191-6.

14. Kira J. Multiple Sclerosis in the Japanese population. Lancet Neurol 2003; 2: 117-127.

15. Singhal BS. Multiple sclerosis-Indian experience. Annals of the Academy of Medicine, Singapore 1985; 14: 32- 6.

16. Cabrera-Gómez JA. Esclerosis Múltiple en el Caribe. Arraigada R, Nogales Gaete, editores. Santiago de Chile; 2002.

17. Yamasaki K, Horiuchi I, Minohara M, et al. HLA-DRB1*0501- associated opticospinal multiple sclerosis: clinical, neuroimaging and immunogenetic studies. Brain 1999; 122: 1689-1696.

18. Jacob ANR, Das K, Boggild M. Exploring the realionship between relapsing mielitis, neuromyelitis optica and multiple sclerosis. J Neurol Neuros Psychiatr 2004; 75: 1225.

19. Katz JD, Romper AH. Progressive necrotic myelopathy: clinical course in 9 patients. Arch Neurol 2000; 57: 355-361.

20. Poppe AY, Lapierre Y, Melancon D, Lowden D, Wardell L, Fullerton LM, et al. Neuromyelitis optica with hypothalamic involvement. Mult Scler 2005; 11: 617-21.

21. Williamson PM. Neuromyelitis optica following infectious mononucleosis. Proc Aust Assoc Neurol 1975; 12: 153-155.

22. Doutlik S, Sblova O, Kryl R, Novak M. Neuromyelitis optica as a parainfectious complication of varicella. Cesk Neurol Neurochir 1975; 38: 238- 242.

23. Blanche P, Díaz E, Gombert B, Sicard D, Rivoal O, Brezin A. Devic's neuromyelitis optica and HIV-1 infection. J Neurol Neurosurg Psychiatry 2000; 68: 795-796.

24. Hagiwara N, Toyoda K, Uwatoko T, Yasumori K, Ibayashi S, Okada Y. Successful high dose glucocorticoid treatment for subacute neuromyelitis optica with systemic lupus erythematosus. Intern Med 2005; 44: 998 1001 .

25. Gibbs AN; Moroney J, Foley-Nolan D, O'Connell PG. Neuromyelitis optica (Devic's syndrome) in systemic lupus erythematosus: a case report. Rheumatology 2002; 41: 470-471.

26. Mochizuki A, Hayashi A, Hisahara S, Shoji S. Steroid- responsive Devic's variant in Sjogren's syndrome. Neurol 2000; 54: 1391-1392.

27. Harada T, Ohashi T, Harada C, et al. A case of bilateral optic neuropathy and recurrent transverse myelopathy associated with perinuclear and anti-neutrophil cytoplasmic antibodies (p-ANCA). J Neuroophthalmol 1997; 17: 254-256.

28. Vernart JC, Cabre P, Smadja D, et al. Recurrent optic neuromyelitis with endocrinopathies: a new syndrome (see comments). Neurol 1997; 48: 58-64.

29. Misu T, Fujihara K, Nakashima I, sato S, Itoyama Y. Intractable hiccup and nausea with periaqueductal lesions in neuromyelitis optica. Neurology 2005; 65: 1479-82.

30. Biliciler S, Uygucgil H, Saip S, Altintas A, Soysal T, Ozdemir SE, et al. Plasmapheresis in multiple sclerosis patients with different indications. Mult Scler 2001; 7 (Suppl 1): 64.

31. Itoyama Y, Saida T, Tashiro K, Sato T, Ohashi Y, Japan TI-bMSCRGi. Japanese multicenter, randomized, double-blind trial of interferon beta$1 \mathrm{~b}$ in relapsing-remitting multiple sclerosis: two year results. Ann Neurol 2000; 48: 487.

32. Dib WJ. Terapia y neuroprotección en la Esclerosis múltiple. Actas del III congreso de Neurociencias de Castilla y León, Valladolid, octubre 2004.

33. Mandler RN, Ahmed W, Dencoff JE. Devic's neuromyelitis optica: a prospective study of seven patients treated with prednisone and azathioprine. Neurol 1998; 51: 1219-20. 